\title{
Prädiktor für eine neurodegenerative Erkrankung
}

Fragestellung: Es wird angenommen, dass die idiopathische Traum-Schlaf-Verhaltensstörung (REM sleep behaviour disorder, iRBD) die Prodromalphase einer Lewy-Körper-Erkrankung darstellt, und die meisten Betroffenen im Verlauf die klinischen Kriterien einer Parkinson-Krankheit (PK), Multisystematrophie (MSA) oder Demenz von Lewy-Körper-Typ (DLB) zeigen werden.

Hintergrund: Die iRBD ist charakterisiert durch einen Verlust der physiologischen Muskelatonie während des REM-Schlafes. Betroffene agieren ihre lebhaften wie auch bedrohlichen Trauminhalte aus.

Patienten und Methodik: Patienten einer iRBD-Kohorte, die 1991 bis 2003 rekrutiert wurden, wurden über weitere sieben Jahre betreut, um den möglichen Beginn einer neurodegenerativen Erkrankung mithilfe standardisierter klinischer Kriterien zu erfassen. Nicht konvertierende iRBD-Patienten wurden mit Dopamintransporter (DAT)-SPECT, transkranieller Parenchymsonografie und Riech-

Iranzo A, Tolosa E, Gelpi E et al. Neurodegenerative disease status and post-mortem pathology in idiopathic rapid-eye-movement sleep behaviour disorder: an observational cohort study. Lancet Neurol 2013; 12: 443-53 tests untersucht. Die Gehirne von drei RBD-Patienten, die klinisch eine PK oder DLB entwickelten, wurden neuropathologisch analysiert.

Ergebnisse: Bis zum Jahr 2012 entwickelten von ur- sprünglich 44 Patienten mit iRBD 36 eine sichere neurodegenerative Erkrankung (16 Patienten mit PK, 14 mit DLB, 1 mit MSA, und fünf mit leichter kognitiver Einschränkung). Eine konversionsfreie Zeit von fünf Jahren wurde von 65,2\% (95\%-Konfidenzintervall [95\%-KI] 50,9-79,5), zehn Jahren von 26,6\% (95\%-KI 12,7-40,5) und 14 Jahren von 7,5\% (95\%-KI -1,9-16,9) der Betroffenen erreicht. Von den restlichen vier iRBD-Patienten zeigten alle reduzierte Signale im striatalen DAT-SPECT, ein Patient zeigte eine Hyperechogenetität der Substantia nigra in der transkraniellen Parenchymsonografie, und zwei hatten eine Hyposmie. Von drei Patienten konnte die klinische Diagnose einer PK und DLB neuropathologisch gesichert werden. In ihren Gehirnen wurden ausgedehnt Lewy-Körper gefunden, und in einem Fall sogar alpha-Synuklein-Aggregate im peripheren autonomen Nervensystem. In allen Gehirnen war ein Verlust von Neuronen und alpha-Synuklein-positive Lewy-Körper und -Neuriten in Hirnstammregionen zu finden, die die Atonie während des REM-Schlafes regulieren.

Schlussfolgerungen: Die meisten iRBD-Patienten entwickelten im Verlauf eine Lewy-Körper-Erkrankung. Die Patienten, die noch keine klinischen Hinweise für eine neurodegenerative Erkrankung boten, zeigten auffällige Befunde wie eine reduzierte DAT-Bindung. Die Autoren folgerten, dass die iRBD die präsymptomatische Phase einer Lewy-Körper-Erkrankung darstellt und als Progressionsmarker bei krankheitsmodifizierenden und neuroprotektiven Therapiestrategien dienen könnte.

\section{- Kommentar von Candan Depboylu, Marburg}

\section{Aufklärung von Betroffenen, Angehörigen und Ärzten ist vordringlich}

Die Arbeit von Iranzo et al. zeigt eindrücklich, dass die Diagnose einer $\mathrm{iRBD}$ einen wichtigen klinischen Risikofaktor darstellt, im Verlauf ein Parkinson-Syndrom, höchstwahrscheinlich eine alpha-Synukleinopathie, zu entwickeln. Die iRBD-Patienten könnten als erste Kandidaten für eine krankheitsmodifizierende Therapiestudie vorgesehen werden. Die mediane Latenz von zwölf bis 14 Jahren von RBD-Beginn bis zur motorischen Manifestation eines Parkinson-Syndroms ermöglicht ein genug langes Fenster für eine präventive Therapie. Dies erfordert jedoch gleichzeitig eine lange Studiendauer - abgesehen von den dann auftretenden Kosten - zur Beurteilung der Effektivität dieser Strategie. Andererseits würde im Rahmen einer solchen Studie das Patientenkollektiv heterogener werden, da die iRBD in unterschiedliche Parkinson-Syndrome (mit unterschiedlicher Prognose) konvertieren würde. Etwa die Hälfte der typischen Parkinson-Patienten haben eine RBD, wobei gerade diese Patienten wiederum klinisch schwerer betroffen zu sein scheinen. Insgesamt ist daher die
RBD mit einer ungünstigen Prognose assoziiert. Um mehr über den Verlauf der iRBD zu erfahren, ist es deshalb wichtig, nach solchen Patienten zu screenen. Die wenigsten Patienten mit einer iRBD suchen jedoch den Arzt auf. Umso dringender erscheint deshalb die Aufklärung von Betroffenen und deren Angehörigen, aber auch von behandelnden Ärzten.

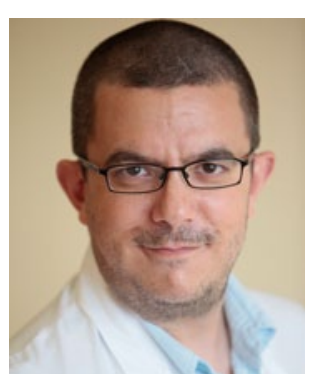

Dr. med. Candan Depboylu, Marburg

Oberarzt der Klinik für Neurologie, Philipps-Universität Marburg E-Mail: depboylu@med.uni-marburg.de 